\title{
Sporotrichosis (Sporothrix schenckii infection) in the New South Wales mid-north coast, 2000-2010
}

\section{Shobini Sivagnanam BSC(Med), MB BS, Infectious Diseases Advanced Trainee \\ Aiveen M Bannan MB BS, FRACP, MPH Infectious Diseases Physician $^{2}$ \\ Sharon C-A Chen FRACP, FRCPA, PhD Infectious Diseases Physician and Microbiologist 1,3 \\ Anna P Ralph MB BS, FRACP, PhD, Infectious Diseases Physician, ${ }^{1,3}$ and Senior Clinical Research Fellow}

1 Centre for Infectious Diseases and Microbiology, Westmead Hospital Sydney, NSW.

2 Port Macquarie Base Hospital, Port Macquarie, NSW.

3 Sydney Institute for Emerging Infectious Diseases and Biosecurity, University of Sydney, Sydney, NSW.

4 Global Health Division Menzies School of Health

Research, Darwin, NT.

Shobini.Sivagnanam@ swahs.health.nsw.gov.au

MJA 2012; 196: 588-590 doi: 10.5694/mjall.10755
$(5$

porotrichosis is a dermatomycosis caused by the dimorphic fungus Sporothrix schenckii. This environmental saprophyte inhabits decaying vegetation, hay, wood, sphagnum moss and soil, and has a ubiquitous global distribution. Infection occurs sporadically in endemic settings or as outbreaks. Although sporotrichosis is rarely associated with dissemination and mortality, ${ }^{1}$ it can cause considerable morbidity owing to delayed diagnosis and inappropriate administration of antibacterial agents. Prompt diagnosis and treatment with antifungal therapy is aided by awareness of classical clues to infection: acquisition in an endemic area; an at-risk occupation or activity such as farming, gardening or bushwalking; an implantation or inoculating injury, such as a penetrating wound from a stick or a cut sustained while handling hay; and characteristic appearance of cutaneous nodules that ulcerate and spread along lymphatic channels, termed "sporotrichoid spread".

The first Australian sporotrichosis case was reported in 1951 in an older Sydney man who had sustained an injury while gardening. ${ }^{2}$ Fifteen further New South Wales cases have been reported, establishing this region as a focus, albeit uncommon, for S. schenckii. ${ }^{2-5}$ It is well recognised in adjacent Queensland, where case reports and series - including an outbreak that affected 16 people and was attributed to mouldy hay ${ }^{6}-$ have amounted to nearly 100 cases since 1956. ${ }^{7-13}$ The most recent Australian report comprised a cluster of 11 cases that were associated with mouldy hay in Western Australia. ${ }^{14}$ However, in NSW, there are no contemporary local data and no cases have been published during the past 26 years.

After observing four cases of sporotrichosis in the NSW mid-north coast, we undertook a retrospective case review to determine the geographical,

\begin{abstract}
Objective: To determine the geographical, epidemiological and clinical features of sporotrichosis (a cutaneous fungal infection caused by Sporothrix schenckii) in the New South Wales mid-north coast.

Design and setting: We undertook a retrospective case review of S. schenckii infections that occurred during the period 2000-2010. Microbiology laboratory staff and medical practitioners in the NSW mid-north coast were contacted to identify cases through database searches and clinical data collection.

Results: Thirty-one cases of S. schenckii infection were identified. Twenty-one occurred in males, the mean age of patients was 59 years, and all cases with data available involved cutaneous disease affecting the limbs (multiple lesions in 18 of 20 cases). Despite an impression of temporal clustering (eight cases in 1 year), this was not statistically significant $(P=0.3)$. Most cases occurred in months with higher rainfall ( 13 of 14 cases with data available). Inoculating events included injuries in gardens, in bushland and on farms, and possible bites. Symptom duration before correct diagnosis ranged from 4 to 30 weeks (median, 8 weeks; data available for 15 cases), unnecessary antibacterial therapy was common ( 13 of 19 cases with data available), and morbidity occurred in the form of recurrence (three cases) and mild itraconazole-induced hepatotoxicity (two cases). In all cases with recorded treatment details, the patients received itraconazole, and the median duration of itraconazole treatment was 24 weeks.

Conclusions: These data highlight that health providers on the NSW mid-north coast should consider sporotrichosis when patients present with characteristic lesions after an inoculating injury sustained from or contaminated by decaying vegetation. The risk may be higher in older people and in wetter months. Fungal culture to confirm the diagnosis needs to be specifically requested. In contrast to outbreaks reported from other states, the epidemiological pattern in NSW appears most consistent with sporadic occurrence in an endemic setting.
\end{abstract}

epidemiological and clinical features of sporotrichosis in this region.

\section{Methods}

Systematic data collection was undertaken to identify sporotrichosis cases in the NSW mid-north coast (Port Macquarie, Wauchope, Kempsey, Taree and surrounds), which has a population of about 153000 . Approval was obtained from the Sydney West Area Health Service Human Research Ethics Committee. Staff from six microbiology laboratories were contacted to perform a database search, where possible, to identify diagnoses of $S$. schenckii infection made during the period 2000-2010. General practitioners, infectious diseases specialists, microbiologists and dermatologists were asked to complete data collection forms. Hospital records were used to obtain additional information.

Data were analysed in Stata version 11.0 (StataCorp, College Station, Tex,
USA), using two-sample $t$ tests, Spearman's rank correlation and nonparametric test for trend. Cases were mapped using the UNSW BioSurveillance Tool (http://www2.chi. unsw.edu.au/biosurveillance/ index.php).

\section{Results}

Three laboratories covered the relevant geographical area and were able to contribute data on cases of $S$. schenckii infection via database searches, and 14 medical practitioners and microbiologists completed data collection forms. During the period 2000-2010, 31 cases of sporotrichosis (29 laboratory-confirmed cases) were identified - an incidence of 2 per 100000 population per annum from multiple locations in a $200 \mathrm{~km}$ coastal zone around Port Macquarie. The mean patient age was 59 years (one case occurred in a child), 21 patients were male, and in all cases 
for which data on lesions were available the patients had cutaneous disease involving one or more limbs (Box 1, Box 2). Inoculating injuries involved vegetation, farming equipment and possible bites; eight involved gardening or farming, and two occurred in previously damaged skin (skin cancer resection and burn scar sites) (Box 1).

Median duration from symptom onset to correct diagnosis was 8 weeks (range, 4-30 weeks). Over the study period, there was no change in duration from symptom onset to correct diagnosis $(P=0.8)$.

Of 19 cases with data available, 13 patients received antibiotics (mostly multiple or prolonged courses) before the correct diagnosis was made. In all 19 cases, the patients were given itraconazole (the currently recommended treatment ${ }^{18}$ ) once the correct diagnosis was made. In two of these cases, patients experienced mild hepatotoxicity - one required treatment interruption and the other required dosage reduction. No data were available on itraconazole serum levels. Median duration of itraconazole treatment was 24 weeks (range, 16-52 weeks).

In 15 of 18 cases with data available, the patients had been cured or their condition had improved at last follow-up. In the remaining three cases, repeat episodes of sporotrichosis were reported. In two of these cases, the sporotrichosis recurred 2 years after symptom onset and the patients were subsequently cured with itraconazole treatment. In the other case, sporotrichosis recurred soon after early cessation of itraconazole treatment at Week 16 due to hepatotoxicity (the lesions had appeared cured before cessation of treatment). This patient was ultimately cured, although no information on the agent used for subsequent treatment was available.

Between zero and eight cases occurred annually, but no year had significantly more cases $(P=0.3)$. Of 14 cases for which month of onset was known, 13 occurred in the 6 months with average monthly rainfall $>120 \mathrm{~mm}$ (Box 3).

\section{Discussion}

Our study shows that the NSW midnorth coast region is an environmental focus for S. schenckii. The incidence of two sporotrichosis cases per 100000 population per annum may be a substantial underestimation because the pathogen is not notifiable. The rural nature of this region and active local agricultural industry provide occupational and recreational means of human exposure to $S$. schenckii.

In contrast to the epidemics reported in $\mathrm{WA}^{14}$ and Qld, ${ }^{6}$ both associated with mouldy hay, these NSW cases arose from a variety of inoculating events. There was an impression of temporal clustering (eight cases in 1 year), but this was not statistically significant. We cannot exclude the possibility that some cases occurred because of a common exposure, but the overall epidemiological pattern appears most consistent with sporadic occurrence in an endemic setting. Sporotrichosis appeared to be associated with wetter months, in keeping with the known habitat of $S$. schenckii; this has been reported previously. ${ }^{15,16}$ The chief limitations of our study are its retrospective design, resulting in missing data and an inability to ensure complete ascertainment of cases, and lack of comparison of NSW mid-north coast rates with rates for other parts of NSW.

Although our small sample size prevents us from drawing major inferences, a number of findings are noteworthy. The preponderance of adult males may reflect their higher likelihood of exposure. One spider and one tick or leech bite were implicated. Although it is unclear how definite these bite histories were, sporotrichosis complicating a spider bite has previously been reported from WA. ${ }^{17}$ Two instances of infection occurred at sites of previous skin injury. Older people appeared to be overrepresented, even though the inoculating injuries sustained are likely to be common across all age groups. Immunosenescence and comorbidities such as diabetes (on which data were not collected) might contribute to risk. Three of the patients in our case series had recurrent disease, but whether these constituted relapse or reinfection is open to speculation. Sporotrichosis recurrence in Australia has not been reported previously, and overseas data suggest that recurrence is uncommon - for
1 Demographic and clinical details of 31 cases of Sporothrix schenckii infection, New South Wales mid-north coast, 2000-2010*

Number ${ }^{\dagger}$

\begin{tabular}{lc}
\hline Age, mean $(95 \% \mathrm{Cl})(n=31)$ & $59(53-66)$ years \\
Males $(n=31)$ & $8(4-30)$ weeks \\
Period before correct diagnosis, & \\
median (range) $(n=15)$ & $24(16-52)$ weeks \\
Duration of itraconazole treatment, & \\
median (range) $(n=10)$ & 2 \\
Lesions $(n=20)$ & 18 \\
Single & \\
Multiple & 14 \\
Site of lesion(s) $(n=20)$ & 6 \\
Upper extremity & \\
Lower extremity & 5 \\
Inoculating events $(n=13)$ & 2 \\
Gardening injury & 12 \\
Scratched on farming equipment & 3 \\
Other farming injury & 3 \\
Injury in bush & 1 \\
Leech or tick bite in swamp & $5^{\ddagger}$ \\
Spider bite & 1 \\
Outcome $(n=18)$ & 1 \\
Cure & \\
Improved & \\
Recurrence & \\
\hline
\end{tabular}

$* n$ indicates number of participants with data available. $†$ Data are number unless otherwise indicated. $¥$ Includes one lesion at a skin cancer resection site. $\oint$ Includes one injury at a burn scar site. Я Probably cured but not followed to completion.

\section{Cutaneous lesions of Sporothrix schenckii infection in a} farmer, showing sporotrichoid spread

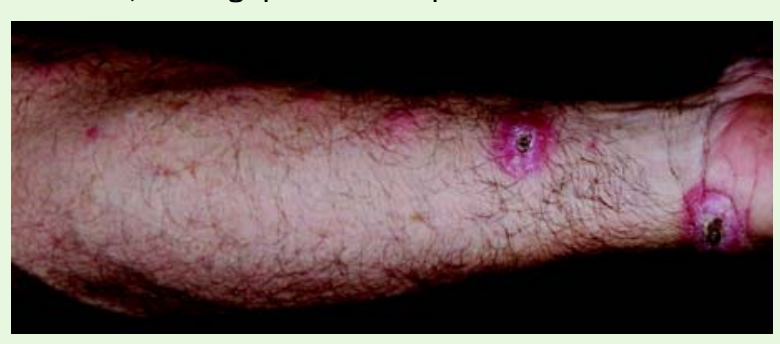

Reproduced with permission, courtesy of Len Moaven.

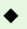

instance, recurrence occurred in one of 45 treated patients in a case series from Peru (48-60 cases per 100 000), a hyperendemic sporotrichosis setting where reinfection is more likely. ${ }^{18}$

Although no cases of disseminated sporotrichosis were identified, the morbidity associated with cutaneous sporotrichosis is evident from long diagnostic delays, inappropriate administration of antibacterial agents, and instances of recurrence and treatment-related complications. Itraconazole is the recommended treatment, with monitoring of serum levels to 
3 Average monthly rainfall (Port Macquarie) and numbers of cases of Sporothrix schenckii infection per month, New South Wales mid-north coast, 2000-2010 $(n=14)$

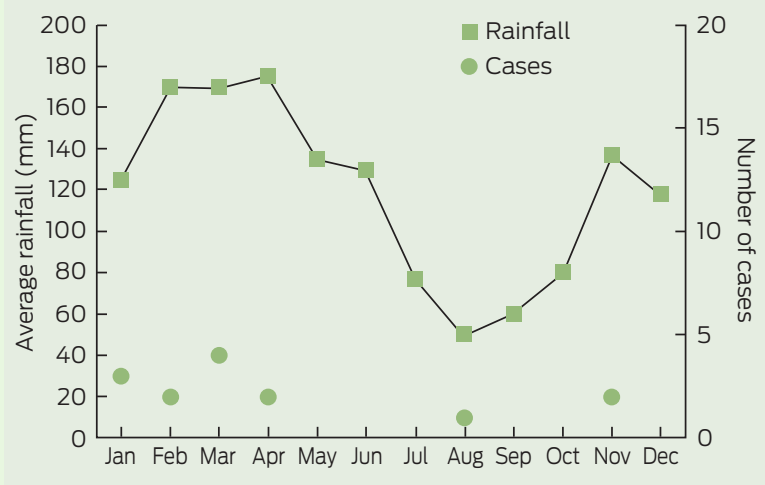

optimise efficacy and safety. ${ }^{19}$ Alternatively, topical saturated solution of potassium iodide or local hyperthermia may be effective. ${ }^{19}$

Early sporotrichosis lesions, especially single lesions (as seen in two cases in our series), can be difficult to distinguish from bacterial infection. Differential diagnoses of lesions showing the classical sporotrichoid spread include infection with atypical mycobacteria (eg, Mycobacterium marinum, Mycobacterium chelonae), Nocardia species and Leishmania species, in the appropriate geographic contexts. Diagnosis can be made either microbiologically by specifically requesting fungal culture (culture on Sabouraud medium at $25^{\circ} \mathrm{C}$ to show the mould form, then on an enrichment media at $37^{\circ} \mathrm{C}$ to show transformation to yeast form), ${ }^{20}$ or histologically (demonstration of gran- ulomas or fungal elements). ${ }^{12}$ Increasing familiarity among health providers might be associated with more prompt diagnoses over time; however, we did not find this, which highlights the need for increased awareness of sporotrichosis in this region.

This report serves to remind local health providers and the general public about sporotrichosis in the NSW mid-north coast. A high index of suspicion is required for diagnosis of this fungal infection when one or more of the characteristic lesions are seen after an inoculating injury sustained from or contaminated by decaying vegetation. The risk may be higher in older people and in wetter months. Fungal culture needs to be specifically requested. Prospective surveillance of sporotrichosis in NSW is needed to provide further clinical and epidemiological data.

Acknowledgements: We thank Len Moaven, Robert Pickles, Ian Hamann, John Ferguson, Catherine Pitman, Raed Simhairi, Robert Clarke, David Cooke, Steve Breathour, Alen Bielich, Roy Scheepers, John Fryatt and Brenton Schuetz for providing data. Anna Ralph is supported by National Health and Medical Research Council Fellowship 1016567 and a Sydney Medical School Foundation grant to the Sydney Emerging Infections and Biosecurity Institute.

Competing interests: No relevant disclosures.

Received 15 Jun 2011, accepted 26 Oct 2011.

1 Silva-Vergara ML, Maneira FR, De Oliveira RM, et al. Multifocal sporotrichosis with meningeal involvement in a patient with AIDS. Med Mycol 2005; 43: 187-190.

2 Robinson CF, Orban TD. A case of regional lymphatic sporotrichosis. Aust J Dermatol 1951; 1 142-144.

3 Barrack BB, Powell RE. Sporotrichosis. Med J Aust 1952; 2: 624-626
4 Durie EB, Frey D, Becke RF. Sporotrichosis: report of a case from Sydney, Australia. Aust J Dermatol 1961; 6: 71-72.

5 Muir DB, Pritchard RC. Sporothrix schenckii incidence in the Sydney region. Australas J Dermatol 1984; 25: 27-28.

6 Conias S, Wilson P. Epidemic cutaneous sporotrichosis: report of 16 cases in Queensland due to mouldy hay. Australas J Dermatol 1998; 39 34-37.

7 Minty CC, Mead M, McCaffrey MF. Sporotrichosis: a case report from Queensland. Med J Aust 1956; 43: 704-705.

8 Mead M, Ridley MF. Sporotrichosis and chromoblastomycosis in Queensland. Med J Aust 1957; 44: 192-197.

9 Robertson D. Report of two cases of sporotrichosis of the face. Australas J Dermatol 1967; 9: 76-82.

10 Auld JC, Beardmore GL. Sporotrichosis in Queensland: a review of 37 cases at the Royal Brisbane Hospital. Australas J Dermatol 1979; 20: 14-22.

11 Beardmore GL. Recalcitrant sporotrichosis: a report of a patient treated with various therapies including oral miconazole and 5-fluorocytosine. Australas J Dermatol 1979; 20: 10-13.

12 Bullpitt P, Weedon D. Sporotrichosis: a review of 39 cases. Pathology 1978; 10: 249-256.

13 Pinn TG. Sporotrichosis in a Queensland bushwalker. Med J Aust 1998; 169: 287.

14 Feeney KT, Arthur IH, Whittle AJ, et al. Outbreak of sporotrichosis, Western Australia. Emerg Infect Dis 2007; 13: 1228-1231.

15 Ghosh A, Chakrabarti A, Sharma VK, et al. Sporotrichosis in Himachal Pradesh (north India). Trans R Soc Trop Med Hyg 1999; 93: 41-45.

16 Mackinnon JE. The dependence on the weather of the incidency of sporotrichosis. Mycopathologia 1948; 4: 367-374.

17 Moaven LD, Altman SA, Newnham AR. Sporotrichosis mimicking necrotising arachnidism [letter]. Med J Aust 1999; 171: 685-686.

18 Pappas PG, Tellez I, Deep AE, et al. Sporotrichosis in Peru: description of an area of hyperendemicity. Clin Infect Dis 2000; 30: 65-70.

19 Kauffman CA, Bustamante B, Chapman SW, Pappas PG; Infectious Diseases Society of America. Clinical practice guidelines for the management of sporotrichosis: 2007 update by the Infectious Diseases Society of America. Clin Infect Dis 2007; 45: 1255-1265.

20 Kauffman CA. Sporotrichosis. Clin Infect Dis 1999; 29: 231-236; quiz 237. 